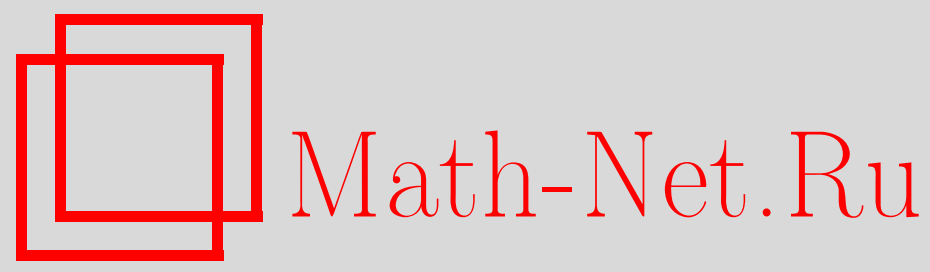

Р. Д. Гецадзе, О функциях Лебега равномерно ограниченных ортонормированных систем, Матем. заметки, 2001, том 69, выпуск 2, 181-193

DOI: https://doi.org/10.4213/mzm494

Использование Общероссийского математического портала Math-Net.Ru подразумевает, что вы прочитали и согласны с пользовательским соглашением http://www.mathnet.ru/rus/agreement

Параметры загрузки:

IP : 52.205 .19 .152

26 апреля 2023 г., $17: 33: 32$

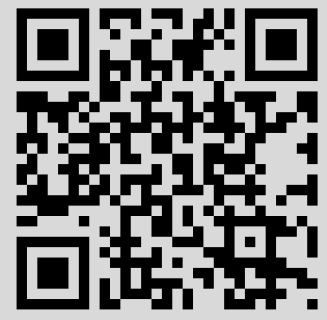




\title{
О ФУНКЦИЯХ ЛЕБЕГА РАВНОМЕРНО ОГРАНИЧЕННЫХ ОРТОНОРМИРОВАННЫХ СИСТЕМ
}

\author{
Р. Д. Гецадзе
}

Установлено дополнение к известному неравенству Олевского о логарифмическом росте функции Лебега произвольной равномерно ограниченной ортонормированной системы на множествах положительной меры. Именно, индекс, для которого функции Лебега имеют рост чуть слабее логарифма, может быть выбран независимо от переменной. Теорема, доказанная в этой статье, усиливает один из результатов, установленных ранее автором.

Библиография: 6 названий.

1. Введение. Известно большое значение функций Лебега в вопросах сходимости и расходимости ортогональных рядов. Фундаментальное неравенство Олевского о росте функций Лебега на множествах положительной меры по общим равномерно ограниченным ортонормированным системам (ОНС) состоит в следующем [1]-[3].

Теорема 1 (А.М. Олевский). Пусть $\left\{\varphi_{n}(x)\right\}_{n=1}^{\infty}-$ произвольная ОНС на [0,1], которая удовлетворяет условию

$$
\left|\varphi_{n}(x)\right| \leqslant M, \quad n=1,2,3, \ldots, \quad x \in[0,1] .
$$

Тогда для любого $n>1$ справедливо неравенство

$$
\mu\left\{x \in[0,1]: \max _{1 \leqslant m \leqslant n} L_{m}(x) \geqslant C \log _{2} n\right\} \geqslant \gamma>0,
$$

где $\mu$ - мера Лебега на [0,1], С и я являются положительными константами, которые зависят только от $M, u$

$$
L_{m}(x)=\int_{0}^{1}\left|\sum_{k=1}^{m} \varphi_{k}(x) \varphi_{k}(\vartheta)\right| d \vartheta, \quad m=1,2, \ldots, \quad x \in[0,1]
$$

- m-я функиия Лебега системы $\left\{\varphi_{n}(x)\right\}_{n=1}^{\infty}$.

В этой статье мы доказьваем теорему, которая дополняет в определенной мере неравенство (2). Мы используем метод, которьй восходит к методу Олевского. Именно, справедлива

Настоящая работа была выполнена во время визита автора в Университет Южной Каролины и была поддержана грантом ОНР НОOО14-91-5-1076. 
Теорема 2. Пусть $\left\{\varphi_{n}(x)\right\}_{n=1}^{\infty}$ - ортонормированная система на $[0,1]$, которая удовлетворяет условию (1). Тогда для кажсдого $n>p_{0}$ существует индекс $m_{n}<n$ такой, что

$$
\mu\left\{x \in[0,1]: L_{m_{n}}(x) \geqslant C_{1} \frac{\log _{2} n}{\log _{2} \log _{2} \log _{2} n}\right\} \geqslant \gamma_{1}>0
$$

где $p_{0}, C_{1}$ и $\gamma_{1}$ - положительные константы, которые зависят только от $M$ uз (1).

Новизна неравенства (4) заключается в том, что индекс $m_{n}$ не зависит от $x$. Более слабое неравенство, чем (4), было установлено нами в [4]. В связи с неравенством (2) мы должны отметить существенный прогресс, достигнутьй в работах [5] и [6].

Мы фактически докажем " $2^{n}$-вариант” теоремы 2 из-за удобства в обозначениях.

Рассмотрим множества

$$
F_{N}=\left\{x \in[0,1]: L_{N}(x) \geqslant \frac{n}{324}\right\}, \quad N=1,2, \ldots
$$

Мы можем допустить, что

$$
\mu F_{N} \leqslant \frac{1}{256 M^{2}} \quad \text { для всех } N=1,2, \ldots, 2^{n+4}
$$

в противном случае теорема 2 уже установлена.

Очевидно, что существует конечная последовательность попарно непересекающихся измеримых множеств $e_{j}^{(n)}, j=1,2, \ldots, q(n)$, такая, что

$$
\bigcup_{j=1}^{q(n)} e_{j}^{(n)}=[0,1]
$$

и для всех $j=1,2, \ldots, q(n)$ и $k=1,2, \ldots, 2^{n+4}$ имеем оценку

$$
\left|\varphi_{k}\left(x_{1}\right)-\varphi_{k}\left(x_{2}\right)\right| \leqslant \frac{1}{2^{3 n}}, \quad x_{1} \in e_{j}^{(n)}, \quad x_{2} \in e_{j}^{(n)}
$$

Пусть также

$$
k(n)=\left[\frac{n}{3 \log _{2} n}\right]
$$

где $[a]$ - целая часть действительного числа $a$.

2. Основная лемма. Теперь мы сформулируем основную лемму. 
ЛЕмма 1. Допустим, что имеет место неравенство (6). Тогда для каждого $n>n_{0}$ существуют конечные последовательности:

а) полохительных целых чисел

$$
m_{0}=2^{n} \leqslant m_{1} \leqslant m_{2} \leqslant \cdots \leqslant m_{k(n)}<2^{n+4}
$$

б) мнохеств

$$
H_{n}^{(p)} \subset[0,1], \quad p=1,2, \ldots, k(n) ;
$$

в) мнохсеств $\Omega_{n}^{(p)}(x) \subset[0,1]$, которые определены для всех $x \in[0,1] u p=1$, $2, \ldots, k(n)$, таких, что выполняются следуюшие условия:

1) числа $m_{p}$ удовлетворяют неравенству

$$
m_{p}-m_{p-1} \leqslant \frac{2^{n+2}}{n^{3(p-1)}} \quad \text { для всех } p=1,2, \ldots, k(n)
$$

2) меры мнохеств $H_{n}^{(p)}$ удовлетворяют неравенству

$$
\mu H_{n}^{(p)} \geqslant \frac{1}{512 M^{2}} \quad \text { для всех } p=1,2, \ldots, k(n)
$$

3) для всех $p=1,2, \ldots, k(n)$ множества $\Omega_{n}^{(p)}(x)$ удовлетворяют условию

$$
\Omega_{n}^{(p)}\left(x_{1}\right)=\Omega_{n}^{(p)}\left(x_{2}\right), \quad x_{1} \in e_{j}^{(n)}, \quad x_{2} \in e_{j}^{(n)}, \quad j=1,2, \ldots, q(n),
$$

u для всех $x \in[0,1]$

$$
\Omega_{n}^{(i)}(x) \cap \Omega_{n}^{(j)}(x)=\varnothing, \quad 1 \leqslant i<j \leqslant k(n) .
$$

Имеют место также следующие неравенства:

$$
\mu \Omega_{n}^{(p)}(x) \leqslant \frac{n^{3(p-1)} n^{2} \log _{2} n}{2^{n}} \quad \text { для всех } p=1,2, \ldots, k(n), x \in[0,1]
$$

и для кажсдого $x \in H_{n}^{(p)}$ имеем

$$
\int_{\Omega_{n}^{(p)}(x)}\left|\sum_{k=1}^{m_{p}} \varphi_{k}(x) \varphi_{k}(\vartheta)\right| d \vartheta \geqslant \frac{1}{386 M^{4}}\left[\frac{\log _{2} n}{3 \log _{2} \log _{2} n}\right]
$$

әде $n_{0}$ зависит только от $M$ из (1). 
3. Доказательство леммы 1. Построение последовательностей $\left\{m_{p}\right\},\left\{H_{n}^{(p)}\right\}$, $\left\{\Omega_{n}^{(p)}(x)\right\}$ (см. (10)-(17)) будет осуществлено индукцией.

Первьй шаг индукции полностью аналогичен $(p+1)$-му шагу. Так что рассмотрим только последний.

Далее допустим, что все шаги осуществлены от первого до $p$-го шага и $p<k(n)$.

3.1. Допушение после $p$-го шага и его анализ. Пусть $F_{p, m}$, где $m>m_{p}$, является множеством всех точек $x$ со свойством: существует измеримое множество $G_{0}(x) \subset[0,1]$ такое, что

$$
\begin{gathered}
\int_{G_{0}(x)}\left|\sum_{k=m_{p}+1}^{m} \varphi_{k}(x) \varphi_{k}(\vartheta)\right| d \vartheta \geqslant \frac{\log _{2} n}{48} \\
\mu G_{0}(x) \leqslant \frac{n^{3 p} n^{2} \log _{2} n}{2^{n}} .
\end{gathered}
$$

Нам понадобится следующая

Лемма 2. Допустим, что некоторое иелое число $\mathrm{m}^{\prime}$ удовлетворяет одновременно следующим условиям:

$$
m_{p} \leqslant m^{\prime} \leqslant m_{p}+\frac{2^{n+2}}{n^{3 p}}, \quad \mu F_{p, m^{\prime}} \geqslant \frac{1}{256 M^{2}} .
$$

Тогда $(p+1)$-й шаг леммы 1 может быть обоснован.

ДокАЗАтЕльство. Действительно, пусть $j$ - индекс такой, что $e_{j}^{(n)} \cap F_{p, m^{\prime}} \neq \varnothing$, $1 \leqslant j \leqslant q(n)$. Тогда для каждого такого $j$ мы выбираем и фиксируем произвольную точку $x_{j}^{(1)}$ из $e_{j}^{(n)} \cap F_{p, m^{\prime}}$. Ясно, что для каждой такой точки существует множество $G_{0}\left(x_{j}^{(1)}\right) \subset[0,1]$, которое удовлетворяет неравенству

$$
\int_{G_{0}\left(x_{j}^{(1)}\right)}\left|\sum_{k=m_{p}+1}^{m^{\prime}} \varphi\left(x_{j}^{(1)}\right) \varphi(\vartheta)\right| d \vartheta \geqslant \frac{\log _{2} n}{48}
$$

и в то же время

$$
\mu G_{0}\left(x_{j}^{(1)}\right) \leqslant \frac{n^{3 p} n^{2} \log _{2} n}{2^{n}} .
$$

Введем множество

$$
\Omega_{n}^{(p+1)}\left(x_{j}^{(1)}\right)=G_{0}\left(x_{j}^{(1)}\right) \backslash \bigcup_{i=1}^{p} \Omega_{n}^{(i)}\left(x_{j}^{(1)}\right) .
$$

Тогда согласно допущению до $p$-го шага леммы 1 мы получаем (cp. (16), (20), (1))

$$
\int_{\bigcup_{i=1}^{p} \Omega_{n}^{(i)}\left(x_{j}^{(1)}\right)}\left|\sum_{k=m_{p}+1}^{m^{\prime}} \varphi_{k}\left(x_{j}^{(1)}\right) \varphi_{k}(\vartheta)\right| d \vartheta \leqslant \frac{4 M^{2} 2^{n}}{n^{3 p}} \sum_{i=1}^{p} \frac{n^{3(i-1)} n^{2} \log _{2} n}{2^{n}} \leqslant \frac{8 M^{2} \log _{2} n}{n} .
$$


Следовательно, имеем для $n>n_{0}$ (cp. (21)-(23))

$$
\begin{gathered}
\int_{\Omega_{n}^{(p+1)}\left(x_{j}^{(1)}\right)}\left|\sum_{k=m_{p}+1}^{m^{\prime}} \varphi_{k}\left(x_{j}^{(1)}\right) \varphi_{k}(\vartheta)\right| d \vartheta \geqslant \frac{\log _{2} n}{49}, \\
\mu \Omega_{n}^{(p+1)}\left(x_{j}^{(1)}\right) \leqslant \frac{n^{3 p} n^{2} \log _{2} n}{2^{n}}
\end{gathered}
$$

Далее, определяем множества $\Omega_{n}^{(p+1)}(x)$ для всех $x \in[0,1]$ на каждом $e_{j}^{(n)}, j=1,2, \ldots$ $\ldots, q(n)$, следуюшими равенствами: $\Omega_{n}^{(p+1)}(x)=\Omega_{n}^{(p+1)}\left(x_{j}^{(1)}\right)$ для всех $x \in e_{j}^{(n)}$, если $e_{j}^{(n)} \cap F_{p, m^{\prime}} \neq \varnothing$, и $\Omega_{n}^{(p+1)}(x)=\varnothing$ для всех $x \in e_{j}^{(n)}$, если $e_{j}^{(n)} \cap F_{p, m^{\prime}}=\varnothing$. Пусть $x \in F_{p, m^{\prime}}$ - произвольная точка. Тогда существует (см. (7)) целое $j_{0}$, зависящее от $x$, такое, что $x \in e_{j_{0}}^{(n)} \cap F_{p, m^{\prime}}$. Мы уже выбрали точку $x_{j_{0}}^{(1)}$ из $e_{j_{0}}^{(n)} \cap F_{p, m^{\prime}}$ такую, что (cм. $(24),(25))$

$$
\int_{\Omega_{n}^{(p+1)}\left(x_{j_{0}}^{(1)}\right)}\left|\sum_{k=m_{p}+1}^{m^{\prime}} \varphi_{k}\left(x_{j_{0}}^{(1)}\right) \varphi_{k}(\vartheta)\right| d \vartheta \geqslant \frac{\log _{2} n}{49},
$$

где

$$
\mu \Omega_{n}^{(p+1)}\left(x_{j_{0}}^{(1)}\right) \leqslant \frac{n^{3 p} n^{2} \log _{2} n}{2^{n}} .
$$

Но тогда по определению множеств $e_{j}^{(n)}$ (см. $\left.(8),(25),(24)\right)$ мы заключаем, что

$$
\int_{\Omega_{n}^{(p+1)}(x)}\left|\sum_{k=m_{p}+1}^{m^{\prime}} \varphi_{k}(x) \varphi_{k}(\vartheta)\right| d \vartheta \geqslant \frac{\log _{2} n}{50}
$$

где (см. (25))

$$
\mu \Omega_{n}^{(p+1)}(x) \leqslant \frac{n^{3 p} n^{2} \log _{2} n}{2^{n}} .
$$

Согласно допущению индукции до $p$-го шага (ср. (14)) и (23) имеем $\Omega_{n}^{(p+1)}(x) \cap \Omega_{n}^{(j)}(x)$ $=\varnothing, 1 \leqslant j \leqslant p, x \in[0,1]$. Далее, определяем множество $H_{n}^{(p+1)}$ и индекс $m_{p+1}$ из леммы 1 . Вводя множество

$$
T_{n}^{(p+1)}=\left\{x \in F_{p, m^{\prime}}: \int_{\Omega_{n}^{(p+1)}(x)}\left|\sum_{k=1}^{m_{p}} \varphi_{k}(x) \varphi_{k}(\vartheta)\right| d \vartheta \geqslant \frac{\log _{2} n}{100}\right\},
$$

положим

$$
\begin{array}{ll}
H_{n}^{(p+1)}=T_{n}^{(p+1)}, \quad m_{p+1}=m_{p}, & \text { если } \mu T_{n}^{(p+1))} \geqslant \frac{1}{512 M_{2}}, \\
H_{n}^{(p+1)}=F_{p, m^{\prime}} \backslash T_{n}^{(p+1)}, \quad m_{p+1}=m^{\prime}, & \text { если } \mu T_{n}^{(p+1)}<\frac{1}{512 M_{2}} .
\end{array}
$$

Лемма 2 (см. (28)-(29)) доказана. 
ЛЕмма 3. Пусть $l$-иелое число такое, что

$$
1 \leqslant l \leqslant\left[\frac{\log _{2} n}{3 \log _{2} \log _{2} n}\right]
$$

иелое $m_{l}^{\prime}$ - произвольный индекс, превосходящийчисло $m_{p}$ из леммы 1 , и $r_{l}^{\prime}$ определено соотношением

$$
r_{l}^{\prime}=m_{l}^{\prime}+\left[\frac{2^{n}}{n^{3 p}\left(\log _{2} n\right)^{3 l}}\right]
$$

Допустим, что для некоторого $x \in[0,1]$ выполнено неравенство

$$
\int_{A_{p, l}}\left|\sum_{k=m_{l}^{\prime}+1}^{r_{l}^{\prime}} \varphi_{k}(x) \varphi_{k}(\vartheta)\right| d \vartheta \geqslant \frac{\log _{2} n}{24}
$$

əде

$$
A_{p, l}=\left\{\vartheta \in[0,1]: \frac{2^{n}}{n^{3 p}\left(\log _{2} n\right)^{3 l} n} \leqslant\left|\sum_{k=m_{l}^{\prime}+1}^{r_{l}^{\prime}} \varphi_{k}(x) \varphi_{k}(\vartheta)\right| \leqslant \frac{2^{n}}{n^{3 p}\left(\log _{2} n\right)^{3 l} \log _{2} n}\right\} .
$$

Tогда

$$
x \in F_{p, m_{l}^{\prime}} \cup F_{p, r_{l}^{\prime}}
$$

ДокАЗАТЕЛЬСтво. Действительно, из (32) и (33) следует, что существует измеримое множество $G_{1}(x)$ со следующими свойствами:

$$
\int_{G_{1}(x)}\left|\sum_{k=m_{l}^{\prime}+1}^{r_{l}^{\prime}} \varphi_{k}(x) \varphi_{k}(\vartheta)\right| d \vartheta=\frac{\log _{2} n}{24}, \quad G_{1}(x) \subset A_{p, l}
$$

Согласно (31) имеем

$$
\mu G_{1}(x) \leqslant \frac{n^{3 p} n^{2} \log _{2} n}{2^{n}} .
$$

Так что получаем или

$$
\int_{G_{1}(x)}\left|\sum_{k=m_{p}+1}^{r_{l}^{\prime}} \varphi_{k}(x) \varphi_{k}(\vartheta)\right| d \vartheta \geqslant \frac{\log _{2} n}{48}
$$

и тогда $x \in F_{p, r_{l}^{\prime}}$, или

$$
\int_{G_{1}(x)}\left|\sum_{k=m_{p}+1}^{m_{l}^{\prime}} \varphi_{k}(x) \varphi_{k}(\vartheta)\right| d \vartheta \geqslant \frac{\log _{2} n}{48}
$$

и тогда $x \in F_{p, m_{l}^{\prime}}$. Лемма 3 доказана. 
3.2. Построение $(p+1)$-го шага леммы 1 . Мы можем допустить, что

$$
\mu F_{p, m} \leqslant \frac{1}{256 M^{2}} \quad \text { для } m_{p}+1 \leqslant m \leqslant m_{p}+\left[\frac{2^{n+2}}{n^{3 p}}\right],
$$

в противном случае достаточно сослаться на лемму 2.

Пусть

$$
r(n)=\left[\frac{\log _{2} n}{3 \log _{2} \log _{2} n}\right] .
$$

Лемма 4. Допустим, что имеет место (35). Тогда для каждого $n>n_{0}$ существуют конечные последовательности:

а) полохительных иелых чисел

$$
m_{0}^{\prime}=m_{p}+1 \leqslant m_{1}^{\prime} \leqslant m_{2}^{\prime} \leqslant \cdots \leqslant m_{r(n)}^{\prime} \leqslant m_{p}+\left[\frac{2^{n+4}}{n^{3 p}}\right]
$$

б) измеримых множеств

$$
h^{(l)} \subset[0,1], \quad l=1,2, \ldots, r(n) ;
$$

в) измеримых множеств $\omega^{(l)}(x) \subset[0,1]$, которые определены для всех $x \in[0,1]$ u $l=1,2, \ldots, r(n)$ таких, что выполнены условия:

1) для чисел $m_{l}^{\prime}$ выполнены неравенства

$$
m_{l}^{\prime}-m_{l-1}^{\prime} \leqslant \frac{2^{n}}{\left(\log _{2} n\right)^{3(l-1)} n^{3 p}} \quad \text { для всех } l=1,2, \ldots, r(n) ;
$$

2) меры множеств $h^{(l)}$ удовлетворяют оценке

$$
\mu h^{(l)} \geqslant \frac{7}{32 M^{2}} \quad \text { для всех } l=1,2, \ldots, r(n) ;
$$

3) для всех $l=1,2, \ldots, r(n)$ выполнено равенство

$$
\begin{aligned}
& \omega^{(l)}\left(x_{1}\right)=\omega^{(l)}\left(x_{2}\right), \quad x_{1} \in e_{j}^{(n)}, \quad x_{2} \in e_{j}^{(n)}, j=1,2, \ldots, r(n), \\
& u \text { для всех } x \in[0,1]
\end{aligned}
$$

$$
\omega^{(i)}(x) \cap \omega^{(j)}(x)=\varnothing, \quad 1 \leqslant i<j \leqslant r(n) .
$$

Имеют место также следующие неравенства:

$$
\mu \omega^{(l)}(x) \leqslant \frac{\left(\log _{2} n\right)^{3(l-1)} n^{3 p} \log _{2} n}{4 M^{2} 2^{n}} \quad \partial_{\text {я }} \text { всех } l=1,2, \ldots, r(n), x \in[0,1]
$$

$u$ для каждого $x \in h^{(l)}$ имеем

$$
\int_{\omega^{(l)}(x)}\left|\sum_{k=m_{p}+1}^{m_{l}^{\prime}} \varphi_{k}(x) \varphi_{k}(\vartheta)\right| d \vartheta \geqslant \frac{1}{20 M^{2}},
$$

әде $n_{0}$ зависит только от $M$ из (1). 
ДокАЗАТЕльСтво. Мы построим последовательности $\left\{m_{l}^{\prime}\right\},\left\{h^{(l)}\right\},\left\{\omega^{(l)}(x)\right\}$ индукцией. Заметим, что конструкция 1-го шага абсолютно аналогична конструкции $(l+1)$-го шага. Так что мы рассмотрим только последний.

Теперь допустим, что все шаги построения осуществлены до $l$-го шага и $l<r(n)$.

Введем целое число

$$
r_{l}^{\prime}=m_{l}^{\prime}+\left[\frac{2^{n}}{n^{3 p}\left(\log _{2} n\right)^{3 l}}\right] .
$$

Ясно, что (см. (10), (45), (37), (39))

$$
2^{n} \leqslant m_{p}+1 \leqslant m_{l}^{\prime} \leqslant r_{l}^{\prime} \leqslant m_{p}+\frac{2^{n+2}}{n^{3 p}} \leqslant 2^{n+4} .
$$

Пусть

$$
k_{l+1}^{\prime}=\left\{x \in[0,1]: \sum_{k=m_{l}^{\prime}+1}^{r_{l}^{\prime}} \varphi_{k}^{2}(x) \geqslant \frac{1}{2}\left[\frac{2^{n}}{n^{3 p}\left(\log _{2} n\right)^{3 l}}\right]\right\} .
$$

Тогда (см. (47), (45))

$$
\mu k_{l+1}^{\prime} \geqslant \frac{1}{2 M^{2}} .
$$

Далее, введем множество

$$
p_{l+1}^{\prime}=k_{l+1}^{\prime} \backslash\left(F_{m_{l}^{\prime}} \cup F_{r_{l}^{\prime}} \cup F_{p, m_{l}^{\prime}} \cup F_{p, r_{l}^{\prime}}\right) .
$$

Согласно допущениям (6) и (35), а также (46), (48) имеем

$$
\mu p_{l+1}^{\prime} \geqslant \frac{1}{2 M^{2}}-\frac{4}{256 M^{2}} \geqslant \frac{7}{16 M^{2}} .
$$

Пусть $i$ - индекс такой, что $e_{i}^{(n)} \cap p_{l+1}^{\prime} \neq \varnothing, 1 \leqslant i \leqslant q(n)$. Для каждого такого $i$ мы выбираем и фиксируем произвольную точку $y_{i}^{(l+1)}$ из $e_{i}^{(n)} \cap p_{l+1}^{\prime}$. Так что мы имеем (cp. (45), (49))

$$
\begin{aligned}
\frac{1}{2}\left[\frac{2^{n}}{n^{3 p}\left(\log _{2} n\right)^{3 l}}\right] \leqslant & \int_{0}^{1}\left(\sum_{k=m_{l}^{\prime}+1}^{r_{l}^{\prime}} \varphi_{k}\left(y_{i}^{(l+1)}\right) \varphi_{k}(\vartheta)\right)^{2} d \vartheta \\
\leqslant & M^{2}\left[\frac{2^{n}}{n^{3 p}\left(\log _{2} n\right)^{3 l}}\right] \int_{B(p, l)}\left|\sum_{k=m_{l}^{\prime}+1}^{r_{l}^{\prime}} \varphi_{k}\left(y_{i}^{l+1}\right) \varphi_{k}(\vartheta)\right| d \vartheta \\
& +\frac{2^{n}}{n^{3 p}\left(\log _{2} n\right)^{3 l} \log _{2} n} \int_{A(p, l)}\left|\sum_{k=m_{l}^{\prime}+1}^{r_{l}^{\prime}} \varphi_{k}\left(y_{i}^{(l+1)}\right) \varphi_{k}(\vartheta)\right| d \vartheta \\
& +\frac{2^{n}}{n^{3 p}\left(\log _{2} n\right)^{3 l} n} \int_{C(p, l)}\left|\sum_{k=m_{l}^{\prime}+1}^{r_{l}^{\prime}} \varphi_{k}\left(y_{i}^{(l+1)}\right) \varphi_{k}(\vartheta)\right| d \vartheta \\
= & I_{1}+I_{2}+I_{3},
\end{aligned}
$$


где

$$
\begin{aligned}
& A_{p, l}=\left\{\vartheta \in[0,1]: \frac{2^{n}}{n^{3 p}\left(\log _{2} n\right)^{3 l} n} \leqslant\left|\sum_{k=m_{l}^{\prime}+1}^{r_{l}^{\prime}} \varphi_{k}\left(y_{i}^{(l+1)}\right) \varphi_{k}(\vartheta)\right| \leqslant \frac{2^{n}}{n^{3 p}\left(\log _{2} n\right)^{3 l} \log _{2} n}\right\},
\end{aligned}
$$

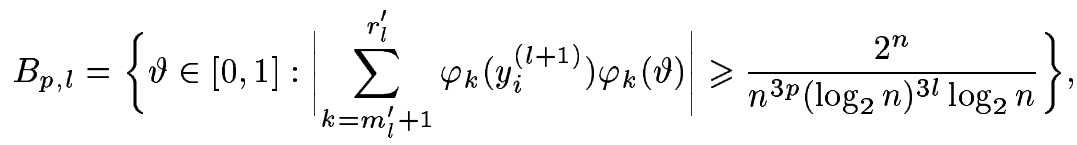

$$
\begin{aligned}
& C_{p, l}=\left\{\vartheta \in[0,1]:\left|\sum_{k=m_{l}^{\prime}+1}^{r_{l}^{\prime}} \varphi_{k}\left(y_{i}^{(l+1)}\right) \varphi_{k}(\vartheta)\right| \leqslant \frac{2^{n}}{n^{3 p}\left(\log _{2} n\right)^{3 l} n}\right\} .
\end{aligned}
$$

Согласно определению множеств $F_{N}(\mathrm{cp} .(5),(3),(46))$ имеем

$$
I_{3} \leqslant \frac{2^{n}}{n^{3 p}\left(\log _{2} n\right)^{3 l} n} \frac{n}{162} \leqslant \frac{2^{n}}{162 n^{3 p}\left(\log _{2} n\right)^{3 l}} .
$$

Из определения множеств $F_{p, m}$, леммы 3 и $(19),(18),(30),(31),(34),(46),(50)$ получаем

$$
I_{2} \leqslant \frac{2^{n}}{24 n^{3 p}\left(\log _{2} n\right)^{3 l}} .
$$

Следовательно, для $n>n_{0}$ выполняется оценка

$$
\int_{B(p, l)}\left|\sum_{k=m_{l}^{\prime}+1}^{r_{l}^{\prime}} \varphi_{k}\left(y_{i}^{(l+1)}\right) \varphi_{k}(\vartheta)\right| d \vartheta \geqslant \frac{1}{4 M^{2}} .
$$

Очевидно, что существует множество $g_{l+1}^{\prime}\left(y_{i}^{(l+1)}\right) \subset B(p, l)$ такое, что

$$
\int_{g_{l+1}^{\prime}\left(y_{i}^{l+1}\right)}\left|\sum_{k=m_{l}^{\prime}+1}^{r_{l}^{\prime}} \varphi_{k}\left(y_{i}^{(l+1)}\right) \varphi_{k}(\vartheta)\right| d \vartheta=\frac{1}{4 M^{2}}
$$

Из (51) имеем

$$
\mu g_{(l+1)}^{\prime}\left(y_{i}^{(l+1)}\right) \leqslant \frac{1}{4 M^{2}} \frac{n^{3 p}\left(\log _{2} n\right)^{3 l} \log _{2} n}{2^{n}}
$$

Пусть

$$
\omega^{(l+1)}\left(y_{i}^{(l+1)}\right)=g_{l+1}^{\prime}\left(y_{i}^{(l+1)}\right) \backslash \bigcup_{j=1}^{l} \omega^{(j)}\left(y_{i}^{(l+1)}\right) .
$$

Тогда согласно допущению индукции (ср. (45), (43)) имеем

$$
\begin{aligned}
& \int_{\bigcup_{j=1}^{l} \omega^{(j)}\left(y_{i}^{(l+1)}\right)\left|\sum_{k=m_{l}^{\prime}+1}^{r_{l}^{\prime}} \varphi_{k}\left(y_{l+1}^{(i)}\right) \varphi_{k}(\vartheta)\right| d \vartheta} \\
& \quad \leqslant \frac{M^{2} 2^{n}}{4 M^{2} n^{3 p}\left(\log _{2} n\right)^{3 l}} \sum_{j=1}^{l} \frac{\left(\log _{2} n\right)^{3(j-1)} n^{3 p} \log _{2} n}{2^{n}} \leqslant \frac{1}{2\left(\log _{2} n\right)^{2}} .
\end{aligned}
$$


Отсюда для $n>n_{0}$ (см. (53)) получаем

$$
\int_{\omega^{(l+1)}\left(y_{i}^{(l+1)}\right)}\left|\sum_{k=m_{l}^{\prime}+1}^{r_{l}^{\prime}} \varphi_{k}\left(y_{i}^{(l+1)}\right) \varphi_{k}(\vartheta)\right| d \vartheta \geqslant \frac{3}{20 M^{2}}
$$

Далее, определяем множества $\omega^{(l+1)}(x)$ для всех $x \in[0,1]$ на каждом $e_{i}^{(n)}, i=1,2, \ldots$ $\ldots, q(n)$, следующими равенствами: $\omega^{(l+1)}(x)=\omega^{(l+1)}\left(y_{i}^{(l+1)}\right)$ для всех $x \in e_{i}^{(n)}$, если $e_{i}^{(n)} \cap p_{l+1}^{\prime} \neq \varnothing$, и $\omega^{(l+1)}(x)=\varnothing$ для всех $x \in e_{i}^{(n)}$, если $e_{i}^{(n)} \cap p_{l+1}^{\prime}=\varnothing$.

Согласно $(8),(7)$ получаем для всех $x \in p_{l+1}^{\prime}$ и $n>n_{0}$ оценку

$$
\int_{\omega^{(l+1)}(x)}\left|\sum_{k=m_{l}^{\prime}+1}^{r_{l}^{\prime}} \varphi_{k}(x) \varphi_{k}(\vartheta)\right| d \vartheta \geqslant \frac{1}{10 M^{2}}
$$

Из (53), (41) очевидно, что для всех $x \in[0,1]$

$$
\omega^{(l+1)}(x) \cap \omega^{(i)}(x)=\varnothing, \quad 1 \leqslant i<l+1 \leqslant r(n) .
$$

Ясно, что

$$
\omega^{(l+1)}\left(x_{1}\right)=\omega^{(l+1)}\left(x_{2}\right), \quad x_{1} \in e_{i}^{(n)}, \quad x_{2} \in e_{i}^{(n)}, \quad i=1,2, \ldots, r(n) .
$$

Введем множество

$$
t^{(l+1)}=\left\{x \in p_{l+1}^{\prime}: \int_{\omega^{(l+1)}(x)}\left|\sum_{k=m_{p}+1}^{m_{l}^{\prime}} \varphi_{k}(x) \varphi_{k}(\vartheta)\right| d \vartheta \geqslant \frac{1}{20 M^{2}}\right\} .
$$

Мы определяем

$$
\begin{array}{ll}
h^{(l+1)}=t^{(l+1)}, \quad m_{l+1}^{\prime}=m_{l}^{\prime}, & \text { если } \mu t^{(l+1)} \geqslant \frac{7}{32 M^{2}}, \\
h^{(l+1)}=p_{l+1}^{\prime} \backslash t^{(l+1)}, \quad m_{l+1}^{\prime}=r_{l}^{\prime}, & \text { если } \mu t^{(l+1)}<\frac{7}{32 M^{2}} .
\end{array}
$$

Очевидно, что (cp. (35)-(46), (50), $(52),(55),(56))(l+1)$-й шаг леммы обоснован и, следовательно, лемма 4 доказана.

Теперь начинаем построение $(p+1)$-го шага леммы 1 , используя лемму 4.

Пусть

$$
e_{n}^{(p+1)}=\left\{x \in[0,1]: \sum_{l=1}^{r(n)} \chi_{l, p}(x) \geqslant \frac{7}{64 M^{2}} r(n)\right\}
$$

где $\chi_{l, p}$ - характеристическая функция множества $h^{(l)}$ из леммы 4 . Очевидно, что (см. (13), (11), (40), (58))

$$
\mu e_{n}^{(p+1)} \geqslant \frac{7}{64 M^{2}} r(n) .
$$


Далее, берем и фиксируем произвольную точку $x$ из $e_{n}^{(p+1)}$. Тогда согласно (58) существует конечная последовательность целых чисел $1 \leqslant l_{1}<\cdots<l_{j^{\prime}(n)} \leqslant r(n)$, зависящая от $x$, такая, что

$$
\begin{gathered}
x \in h^{\left(l_{i}\right)}, \quad i=1,2, \ldots, j^{\prime}(n), \\
j^{\prime}(n) \geqslant \frac{7}{64 M^{2}} r(n) .
\end{gathered}
$$

Имея в виду (39), мы заключаем, что для всех $i=1,2,3, \ldots, j^{\prime}(n)$

$$
\begin{aligned}
m_{r(n)}^{\prime}-m_{l_{i}}^{\prime}= & \sum_{s=l_{i}+1}^{r(n)}\left(m_{s}^{\prime}-m_{s-1}^{\prime}\right) \leqslant \sum_{s=l_{i}+1}^{r(n)} \frac{2^{n}}{\left(\log _{2} n\right)^{3(s-1)} n^{3 p}} \leqslant \frac{2^{n+1}}{\left(\log _{2} n\right)^{3 l_{i} n^{3 p}}} \\
& \int_{\omega^{\left(l_{i}\right)(x)}}\left|\sum_{k=m_{p}+1}^{m_{l_{i}}^{\prime}} \varphi_{k}(x) \varphi_{k}(\vartheta)\right| d \vartheta \geqslant \frac{1}{20 M^{2}} .
\end{aligned}
$$

Согласно (1), (60)-(63), (43), (42) получаем, что

$$
\begin{aligned}
& \int_{\bigcup_{l=1}^{r(n)} \omega^{(l)}(x)}\left|\sum_{k=m_{p}+1}^{m_{r(n)}^{\prime}} \varphi_{k}(x) \varphi_{k}(\vartheta)\right| d \vartheta \\
& \geqslant \sum_{i=1}^{j^{\prime}(n)} \int_{\omega^{\left(l_{i}\right)}(x)}\left|\sum_{k=m_{p}+1}^{m_{r(n)}^{\prime}} \varphi_{k}(x) \varphi_{k}(\vartheta)\right| d \vartheta \\
& \geqslant \sum_{i=1}^{j^{\prime}(n)} \int_{\omega^{\left(l_{i}\right)}(x)}\left|\sum_{k=m_{p}+1}^{m_{l_{i}}^{\prime}} \varphi_{k}(x) \varphi_{k}(\vartheta)\right| d \vartheta-\sum_{i=1}^{j^{\prime}(n)} \int_{\omega^{\left(l_{i}\right)}(x)}\left|\sum_{k=m_{l_{i}}^{\prime}+1}^{m_{r(n)}^{\prime}} \varphi_{k}(x) \varphi_{k}(\vartheta)\right| d \vartheta \\
& \geqslant \frac{j^{\prime}(n)}{20 M^{2}}-M^{2} \sum_{i=1}^{j^{\prime}(n)} \mu \omega^{\left(l_{i}\right)}(x)\left(m_{r(n)}^{\prime}-m_{l_{i}}^{\prime}\right) \\
& \geqslant \frac{j^{\prime}(n)}{20 M^{2}}-2 M^{2} \sum_{i=1}^{j^{\prime}(n)} \frac{\log _{2} n\left(\log _{2} n\right)^{3\left(l_{i}-1\right)}}{4 M^{2}\left(\log _{2} n\right)^{3 l_{i}}} \geqslant \frac{1}{192 M^{4}}\left[\frac{\log _{2} n}{3 \log _{2} \log _{2} n}\right] .
\end{aligned}
$$

Следовательно, мы построили множество $e_{n}^{(p+1)}$ такое, что имеет место неравенство (59), и для всех $x \in e_{n}^{(p+1)}$ имеем

$$
\int_{G_{n}^{(p+1)}(x)}\left|\sum_{k=m_{p}+1}^{m_{r(n)}^{\prime}} \varphi_{k}(x) \varphi_{k}(\vartheta)\right| d \vartheta \geqslant \frac{1}{192 M^{4}}\left[\frac{\log _{2} n}{3 \log _{2} \log _{2} n}\right]
$$

где

$$
G_{n}^{(p+1)}(x)=\bigcup_{l=1}^{r(n)} \omega^{(l)}(x)
$$


Заметим, что (см. (43), (36), (65)) для всех $x \in[0,1]$ вьполнена оценка

$$
\mu G_{n}^{(p+1)}(x) \leqslant \sum_{l=1}^{r(n)} \frac{\left(\log _{2} n\right)^{3(l-1)} n^{3 p} \log _{2} n}{4 M^{2} 2^{n}} \leqslant \frac{n^{3 p} n \log _{2} n}{4 M^{2} 2^{n}} .
$$

Далее, определяем множество $\Omega_{n}^{(p+1)}(x)$ в лемме 1 следуюшим образом:

$$
\Omega_{n}^{(p+1)}(x)=G_{n}^{(p+1)}(x) \backslash \bigcup_{i=1}^{p} \Omega_{n}^{(i)}(x)
$$

Из допушения индукции в лемме 1 до $p$-го шага (см. (16)) имеем

$$
\begin{aligned}
\int_{\bigcup_{i=1}^{p} \Omega_{n}^{(i)}(x)}\left|\sum_{k=m_{p}+1}^{m_{r(n)}^{\prime}} \varphi_{k}(x) \varphi_{k}(\vartheta)\right| d \vartheta & \leqslant M^{2}\left(m_{r(n)}^{\prime}-m_{p}\right) \sum_{i=1}^{p} \mu \Omega_{n}^{(i)}(x) \\
& \leqslant \frac{M^{2} 2^{n}}{n^{3 p}} \sum_{i=1}^{p} \frac{n^{3(i-1)} n^{2} \log _{2} n}{2^{n}} \leqslant \frac{2 M^{2} \log _{2} n}{n} .
\end{aligned}
$$

Мы заключаем из (66), (64), что для $n>n_{0}$ и для всех $x \in e_{n}^{(p+1)}$ вьполнена оценка

$$
\int_{\Omega_{n}^{(p+1)}(x)}\left|\sum_{k=m_{p}+1}^{m_{r(n)}^{\prime}} \varphi_{k}(x) \varphi_{k}(\vartheta)\right| d \vartheta \geqslant \frac{1}{193 M^{4}}\left[\frac{\log _{2} n}{3 \log _{2} \log _{2} n}\right] .
$$

Введем множество

$$
Q_{n}^{(p+1)}=\left\{x \in e_{n}^{(p+1)}: \int_{\Omega_{n}^{(p+1)}(x)}\left|\sum_{k=1}^{m_{p}} \varphi_{k}(x) \varphi_{k}(\vartheta)\right| d \vartheta \geqslant \frac{1}{386 M^{4}}\left[\frac{\log _{2} n}{3 \log _{2} \log _{2} n}\right]\right\} .
$$

Далее, определяем множество $H_{n}^{(p+1)}$ и индекс $m_{p+1}$ из леммы 1 . Пусть $H_{n}^{(p+1)}=Q_{n}^{(p+1)}$ и $m_{p+1}=m_{p}$, если $\mu Q_{n}^{(p+1)} \geqslant 7 /\left(128 M^{2}\right)$, и $H_{n}^{(p+1)}=e_{n}^{(p+1)} \backslash Q_{n}^{(p+1)}$ и $m_{p+1}=m_{r(n)}^{\prime}$, если $\mu Q_{n}^{(p+1))}<7 /\left(128 M^{2}\right)$. Лемма 1 (см. (65), (66)) доказана.

4. Доказательство теоремы 2. Введем множество

$$
E_{n}=\left\{x \in[0,1]: \sum_{p=1}^{k(n)} \chi_{l, p}^{(1)}(x) \geqslant \frac{1}{1024 M^{2}} k(n)\right\}
$$

где $\chi_{l, p}^{(1)}$ - характеристическая функция множества $H_{n}^{(p)}$ из леммы 1.

Ясно, что

$$
\mu E_{n} \geqslant \frac{1}{1024 M^{2}}
$$


Далее, фиксируем произвольную точку $x$ из $E_{n}$. Тогда (см. (67)) существует конечная последовательность $1 \leqslant p_{1}<\cdots<p_{j(n)} \leqslant k(n)$ индексов, зависящих от $x$, такая, что $x \in H_{n}^{\left(p_{i}\right)}, i=1,2, \ldots, j(n)$, и

$$
j(n) \geqslant \frac{1}{1024 M^{2}} k(n) .
$$

Далее, пусть $N_{n}=m_{k(n)}$. Учитьвая (12), для всех $i=1,2, \ldots, k(n)$ имеем

$$
N_{n}-m_{p_{i}}=\sum_{p=p_{i}}^{k(n)-1}\left(m_{p+1}-m_{p}\right) \leqslant \sum_{p=p_{i}}^{k(n)-1} \frac{2^{n+2}}{n^{3 p}} \leqslant \frac{2^{n+3}}{n^{3 p_{i}}} .
$$

Кроме того,

$$
N_{n}=m_{0}+\sum_{p=0}^{k(n)-1}\left(m_{p+1}-m_{p}\right) \leqslant 2^{n}+2^{n+2} \sum_{p=0}^{k(n)-1} \frac{1}{n^{3 p}} \leqslant 2^{n+4} .
$$

Согласно (68), (9), (15)-(17) очевидно, что

$$
\begin{aligned}
\int_{0}^{1}\left|\sum_{k=1}^{N_{n}} \varphi_{k}(x) \varphi_{k}(\vartheta)\right| d \vartheta & \geqslant \sum_{i=1}^{j(n)} \int_{\Omega_{n}^{\left(p_{i}\right)}(x)}\left|\sum_{k=1}^{N_{n}} \varphi_{k}(x) \varphi_{k}(\vartheta)\right| d \vartheta \\
& \geqslant \sum_{i=1}^{j(n)} \int_{\Omega_{n}^{\left(p_{i}\right)}(x)}\left|\sum_{k=1}^{m_{p_{i}}} \varphi_{k}(x) \varphi_{k}(\vartheta)\right| d \vartheta \\
& -\sum_{i=1}^{j(n)} \int_{\Omega_{n}^{\left(p_{i}\right)}(x)}\left|\sum_{k=m_{p_{i}}+1}^{N_{n}} \varphi_{k}(x) \varphi_{k}(\vartheta)\right| d \vartheta \\
\geqslant & \frac{C_{2} j(n) \log _{2} n}{\log _{2} \log _{2} n}-\sum_{i=1}^{j(n)} M^{2}\left(N_{n}-m_{p_{i}}\right) \mu \Omega_{n}^{\left(p_{i}\right)}(x) \geqslant \frac{C_{3} n}{\log _{2} \log _{2} n}
\end{aligned}
$$

для некоторых констант $C_{2}$ и $C_{3}$, которые зависят только от $M$ из (1).

Теорема 2 доказана.

\section{СПИСОК ЦИТИРОВАННОЙ ЛИТЕРАТУРЫ}

[1] Олевский А. М. Ряды Фурье непрерьвных функций по ограниченньм ортонормальным системам // Изв. АН СССР. Сер. матем. 1966. Т. 30. С. 387-432.

[2] Олевский А. М. О порядке роста функции Лебега ограниченных ортонормированных систем // Докл. АН СССР. Сер. матем. 1967. Т. 176. С. 1247-1250.

[3] Olevsky A. M. Fourier Series with Respect to General Orthogonal Systems. Berlin: SpringerVerlag, 1975.

[4] Гецадзе Р. Д. О расходимости по мере кратных ортогональных рядов Фурье // Тр. МИАН. 1989. Т. 190 . С. $75-87$.

[5] Бочкарев С. В. Логарифмический рост средних арифметических от функции Лебега ограниченньх ортонормированных систем // Докл. АН СССР. 1975. Т. 233. С. 16-19.

[6] Кашин Б. С., Саакян А. А. Ортогональные ряды. М.: Наука, 1984. 